\title{
BENDING SOLUTION OF HIGH-ORDER REFINED SHEAR DEFORMATION THEORY FOR RECTANGULAR COMPOSITE PLATES
}

\author{
LIU PING $†$, ZHANG YONGWEI $\ddagger$ and ZHANG KAIDA $\dagger$ \\ +Department of Aircraft, Northwestern Polytechnical University, Xi'an, 710072. \\ People's Republic of China \\ \$Basic Research Division, Institute of Mechanics, Chinese Academy of Sciences, Beijing, \\ 100080, People's Republic of China
}

(Received 8 July 1993; in revised form 28 February 1994)

\begin{abstract}
A new high-order refined shear deformation theory based on Reissner's mixed variational principle in conjunction with the state-space concept is used to determine the deflections and stresses for rectangular cross-ply composite plates. A zig-zag shaped function and Legendre polynomials are introduced to approximate the in-plane displacement distributions across the plate thickness. Numerical results are presented with different edge conditions, aspect ratios, lamination schemes and loadings. A comparison with the exact solutions obtained by Pagano and the results by Khdeir indicates that the present theory accurately estimates the in-plane responses.
\end{abstract}

\section{INTRODUCTION}

Three-dimensional elasticity solutions for the bending of simply supported thick orthotropic rectangular plates and laminates were obtained by Srinivas and Rao (1970), Srinivas et al. (1970), Hussainy and Srinivas (1975) and Pagano (1970). The Navier solution of simply supported rectangular plates was developed by Whitney and Leissa (1969) for classical laminate theory, Whitney and Pagano (1970), Bert and Chen (1978) and Reddy and Chao (1981) for the first-order shear deformation (i.e. the Reissner-Mindlin plate) theory, and by Reddy (1984a,b) and Reddy and Phan (1985) for refined shear deformation theories. The Lévy type solutions were developed by Reddy et al. (1987) for symmetric laminates with different combinations of free, clamped and simply supported boundary conditions by using the first-order shear deformation theory. Khdeir et al. (1987) later extended Reddy's work by using refined shear deformation theory.

Murakami (1986) proposed an improved in-plane response theory based on Reissner's (1984) mixed variational principle and applied it to cylindrical bending problem of laminated plates, the improvement was achieved by including a zig-zag shaped function to approximate the in-plane displacements across the thickness. However, this theory cannot exactly describe the deformation of the anti-symmetric and irregular laminated plates.

Based upon Murakami's theory, Legendre polynomials are introduced in the displacement field and the transverse normal strain is also included in present theory so that the in-plane displacement distribution for arbitrary laminated configurations can be determined exactly. The advantage of using Reissner's mixed variation principle is that it automatically yields the appropriate shear correction factors for the transverse shear constitutive equations. Other attractive features of the present theory are : (1) the continuity condition of transverse shear stresses at the interfaces is satisfied; (2) the effects of the transverse shear and transverse normal strains are accounted; (3) the number of equations remains unchanged as the number of layers increases.

The accuracy of the present theory is examined by applying it to bending problem of rectangular laminates with two opposite edges simply supported and the remaining edges subject to a combination of free, simply supported and clamped boundary conditions. Different aspect ratios, lamination schemes and loadings are considered. The state-space concept is used to solve the ordinary differential equations. 


\section{GOVERNING EQUATIONS}

Consider an $N$-layer laminated composite plate, shown in Fig. 1. The following notation, ()$^{(k)}, k=1,2 \ldots N$, will designate quantities associated with the $k$ th layer. The thickness of each layer is $n^{(k)} h$. Unless otherwise specified, the usual Cartesian indicial notation is employed where Latin and Greek indices range from 1 to 3 and 1 to 2 , respectively. Repeated indices imply the summation convection and ()$_{, i}$ denotes partial differentiation with respect to $x_{i}$.

Constitutive equations for orthotropic layers (Murakami, 1986):

$$
\begin{aligned}
& {\left[\begin{array}{l}
\sigma_{11} \\
\sigma_{22} \\
\sigma_{12}
\end{array}\right]^{(k)}=\left[\begin{array}{ccc}
\bar{c}_{11} & \bar{c}_{12} & 0 \\
\bar{c}_{12} & \bar{c}_{22} & 0 \\
0 & 0 & c_{66}
\end{array}\right]^{(k)}\left[\begin{array}{c}
e_{11} \\
e_{22} \\
2 e_{12}
\end{array}\right]^{(k)}+\left[\begin{array}{c}
\frac{c_{13}}{c_{33}} \\
\frac{c_{23}}{c_{33}} \\
0
\end{array}\right]^{(k)} \sigma_{33}^{(k)}} \\
& {\left[\begin{array}{l}
e_{33} \\
2 e_{23} \\
2 e_{31}
\end{array}\right]^{(k)}=-\left[\begin{array}{ccc}
\frac{c_{13}}{c_{33}} & \frac{c_{23}}{c_{33}} & 0 \\
0 & 0 & 0 \\
0 & 0 & 0
\end{array}\right]^{(k)}\left[\begin{array}{c}
e_{11} \\
e_{22} \\
2 e_{12}
\end{array}\right]^{(k)}+\left[\begin{array}{ccc}
\frac{1}{c_{33}} & 0 & 0 \\
0 & \frac{1}{c_{44}} & 0 \\
0 & 0 & \frac{1}{c_{55}}
\end{array}\right]^{(k)}\left[\begin{array}{l}
\sigma_{33} \\
\sigma_{23} \\
\sigma_{31}
\end{array}\right]^{(k)} ;}
\end{aligned}
$$

strain-displacement relations

$$
e_{i j}^{(k)}=\frac{1}{2}\left(u_{i, j}^{(k)}+u_{j, i}^{(k)}\right)
$$

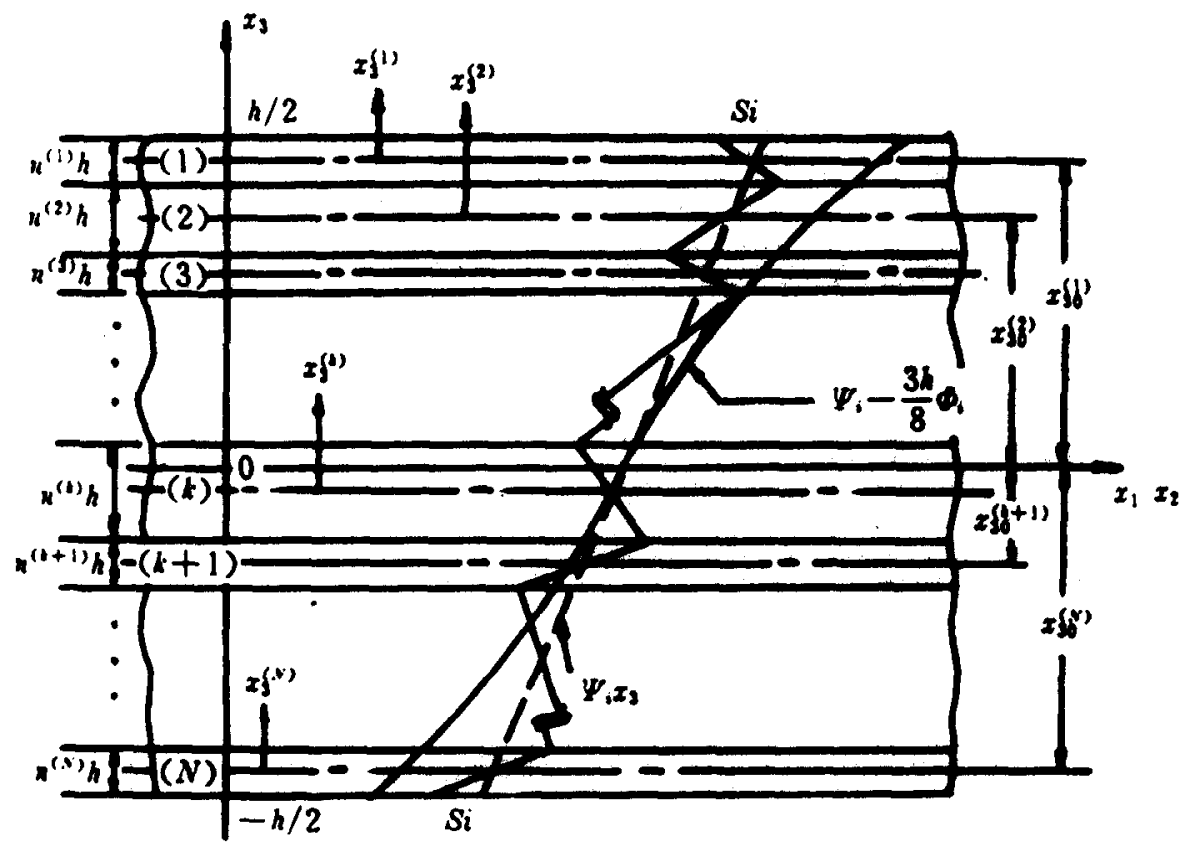

Fig. 1. Plate geometry coordinate system and trial in-plane displacements. 
interface continuity conditions

$$
u_{i}^{(k)}=u_{i}^{(k+1)} \quad \text { and } \quad \sigma_{3 i}^{(k)}=\sigma_{3 i}^{(k+1)} \quad k=1,2 \ldots N-1
$$

upper and lower surface stress conditions

$$
\begin{aligned}
& \sigma_{3 i}^{(1)}=T_{i}^{+} \quad \text { on } \quad x_{3}=\frac{h}{2} \\
& \sigma_{3 i}^{(N)}=T_{i}^{-} \text {on } x_{3}=-\frac{h}{2} \text {. }
\end{aligned}
$$

Reissner's mixed variational principle was applied to $N$-layer composite plate whose middle surface occupies a domain $D$ in the $x_{1}, x_{2}$-plane :

$$
\begin{array}{r}
\iint_{D}\left[\sum_{k} \int_{A^{(k)}}\left\{\delta e_{i j}^{(k)} \sigma_{i j}^{(k)}+\left[u_{\alpha, 3}^{(k)}+u_{3, \alpha}^{(k)}-2 e_{3 \alpha}^{(k)}(\ldots)\right] \delta \tau_{3 \alpha}^{(k)}+\left[u_{3,3}^{(k)}-e_{33}^{(k)}(\ldots)\right] \delta \tau_{33}^{(k)}\right\} \mathrm{d} x_{3}\right] \mathrm{d} x_{1} \mathrm{~d} x_{2} \\
=\int_{\partial D_{T}}\left[\sum_{k} \int_{A^{(k)}} \delta u_{i}^{(k) t} T_{i}^{(k)} \mathrm{d} x_{3}\right] \mathrm{d} s+\iint_{D}\left[\delta u_{i}^{(1)}\left(x_{1}, x_{2}, \frac{h}{2}\right) T_{i}^{+}\right. \\
\left.-\delta u_{i}^{(N)}\left(x_{1}, x_{2},-\frac{h}{2}\right) T_{i}^{-}\right] \mathrm{d} x_{1} \mathrm{~d} x_{2}
\end{array}
$$

where $\partial D_{T}$ denotes the boundary of $D$ with outward normal $v_{\alpha}$ on which tractions $T_{i}$ are specified and $\boldsymbol{A}^{(k)}$ represents the $x_{3}$-domain occupied by the $k$ th layer. Also $\tau_{3 i}^{(k)}$ denote the approximate transverse stresses and $e_{3 i}^{(k)} \ldots$ implies the appropriate right-hand side of eqn (1b).

The high-order laminated plate theory, which takes into account the effect of transverse shear strains, is obtained by including the Legendre polynomials of order $n=1,2,3$, with respect to the $x_{3}$-coordinate to a zig-zag in-plane displacement variation of amplitude $S_{i}\left(x_{1}\right.$, $x_{2}$ ) across the plate thickness.

The appropriate trial functions used in connection with Reissner's mixed variational principle eqn (5) are taken to be:

(a) trial displacement field (see Fig. 1)

$$
\begin{aligned}
u_{i}^{(k)}\left(x_{1}, x_{2}, x_{3}\right)=U_{i}\left(x_{1}, x_{2}\right)+ & \frac{h}{2} \Psi_{i}\left(x_{1}, x_{2}\right) P_{1}(\zeta)+S_{i}\left(x_{1}, x_{2}\right)(-1)^{k} \frac{2}{n^{(k)} h} x_{3}^{(k)} \\
& +\left(\frac{h}{2}\right)^{2} \xi_{i}\left(x_{1}, x_{2}\right) P_{2}(\zeta)+\left(\frac{h}{2}\right)^{3} \Phi_{i}\left(x_{1}, x_{2}\right) P_{3}(\zeta),
\end{aligned}
$$

where $\zeta \equiv 2 x_{3} / h$ and $P_{n}(\zeta)$ are the Legendre polynomials of order $n$ and $\Phi_{3} \equiv 0, x_{3}^{(k)}$ is a local $x_{3}$-coordinate system with its origin at the center $x_{30}^{(k)}$ of the $k$ th layer, i.e.

$$
x_{3}^{(k)} \equiv x_{3}-x_{30}^{(k)}
$$

(b) trial transverse and normal stresses

$$
\begin{aligned}
\tau_{3 \alpha}^{(k)}\left(x_{1}, x_{2}, x_{3}\right)= & Q_{\alpha}^{(k)}\left(x_{1}, x_{2}\right) F_{1}(z)+R_{\alpha}^{(k)}\left(x_{1}, x_{2}\right) F_{2}(z) \\
& +J_{\alpha}^{(k)}\left(x_{1}, x_{2}\right) F_{3}(z)+\left[T_{\alpha}^{(k-1)}\left(x_{1}, x_{2}\right)+T_{\alpha}^{(k)}\left(x_{1}, x_{2}\right)\right] F_{4}(z) \\
& +\left[T_{\alpha}^{(k-1)}\left(x_{1}, x_{2}\right)-T_{\alpha}^{(k)}\left(x_{1}, x_{2}\right)\right] F_{5}(z)
\end{aligned}
$$




$$
\begin{aligned}
\tau_{33}^{(k)}\left(x_{1}, x_{2}, x_{3}\right)= & Q_{3}^{(k)}\left(x_{1}, x_{2}\right) F_{1}(z)+R_{3}^{(k)}\left(x_{1}, x_{2}\right) F_{6}(z) \\
& +J_{3}^{(k)}\left(x_{1}, x_{2}\right) F_{3}(z)+I_{3}^{(k)}\left(x_{1}, x_{2}\right) F_{7}(z) \\
& +\left[T_{3}^{(k-1)}\left(x_{1}, x_{2}\right)+T_{3}^{(k)}\left(x_{1}, x_{2}\right)\right] F_{4}(z) \\
& +\left[T_{3}^{(k-1)}\left(x_{1}, x_{2}\right)-T_{3}^{(k)}\left(x_{1}, x_{2}\right)\right] F_{8}(z),
\end{aligned}
$$

where

$$
\begin{aligned}
& F_{1}(z)=\frac{5}{n^{(k)} h}\left(21 z^{4}-\frac{15}{2} z^{2}+\frac{9}{16}\right), F_{2}(z)=\frac{-30}{\left(n^{k} h\right)^{2}}\left(4 z^{3}-z\right) \\
& F_{3}(z)=\frac{-105}{\left(n^{(k)} h\right)^{3}}\left(20 z^{4}-6 z^{2}+\frac{1}{4}\right), F_{4}(z)=35 z^{4}-\frac{15}{2} z^{2}+\frac{3}{16} \\
& F_{5}(z)=10 z^{3}-\frac{3}{2} z, F_{6}(z)=\frac{105}{\left(n^{k} h\right)^{2}}\left(36 z^{5}-14 z^{3}+\frac{5}{4} z\right) \\
& F_{7}(z)=\frac{-315}{\left(n^{(k)} h\right)^{4}}\left(112 z^{5}-40 z^{3}+3 z\right), F_{8}(z)=126 z^{5}-35 z^{3}+\frac{15}{8} z \\
& z \equiv \frac{x_{3}^{(k)}}{n^{(k)} h} \quad-\frac{1}{2} \leqslant z \leqslant \frac{1}{2} .
\end{aligned}
$$

Also

$$
\begin{aligned}
\left(Q_{i}^{(k)}, R_{i}^{(k)}, J_{i}^{(k)}\right) & \equiv \int_{A^{(k)}}\left(1, x_{3}^{(k)}, x_{3}^{(k) 2}\right) \tau_{3 i}^{(k)} \mathrm{d} x_{3} \\
I_{3}^{(k)} & \equiv \int_{A^{(k)}} x_{3}^{(k) 3} \tau_{33}^{(k)} \mathrm{d} x_{3} .
\end{aligned}
$$

In eqn (8), $T_{i}^{(k-1)}$ and $T^{(i)}$ are the values of $\tau_{3 i}^{(k)}$ at the top and bottom surfaces of the $k$ th layer, respectively, from eqn (4)

$$
T_{i}^{(0)}=T_{i}^{+} \quad \text { and } \quad T_{i}^{(N)}=T_{i}^{-} .
$$

The functions $F_{i}(z), i=1 \ldots 8$ are obtained by first noting that eqn (6) yields cubic variations across the plate thickness of in-plane stresses. From the equilibrium equations (i.e. $\sigma_{j i, j}^{(k)}=0$ ), transverse stresses $\tau_{3 \alpha}^{(k)}$ and $\tau_{33}^{(k)}$ may, as a result, be represented by polynomials of degree 4 and 5 in $z$, respectively. Their corresponding coefficients are then computed by using eqns $(10 \mathrm{a}, \mathrm{b})$. This yields the functions $F_{i}(z)$.

Substituting eqns (6) and (8) into eqn (5), using Gauss' theorem and the orthogonality relationship of the Legendre polynomials, one obtains laminated plate equations :

(a) equilibrium equations

$$
\begin{aligned}
N_{\alpha i, \alpha}+T_{i}^{+}-T_{i}^{-} & =0 \\
M_{\alpha i, \alpha}-N_{3 i}+\frac{h}{2}\left(T_{i}^{+}+T_{i}^{-}\right) & =0 \\
Z_{\alpha i, \alpha}-K_{3 i}-\left[T_{i}^{+}-(-1)^{N} T_{i}^{-}\right] & =0 \\
L_{\alpha i, \alpha}-3 M_{3 i}+\frac{h^{2}}{4}\left(T_{i}^{+}-T_{i}^{-}\right) & =0
\end{aligned}
$$




$$
P_{\beta \alpha, \beta}-\left(5 L_{3 \alpha}+\frac{h^{2}}{4} N_{3 \alpha}\right)+\frac{h^{3}}{8}\left(T_{\alpha}^{+}+T_{\alpha}^{-}\right)=0
$$

$\left[N_{\alpha \beta}, M_{\alpha \beta}, Z_{\alpha \beta}, L_{\alpha \beta}, P_{\alpha \beta}\right] \equiv \sum_{k=1}^{N} \int_{A^{(k)}}\left[1, \frac{h}{2} P_{1}(\zeta),(-1)^{k} \frac{2 x_{3}^{(k)}}{n^{(k)} h}\right.$,

$$
\left.\left(\frac{h}{2}\right)^{2} P_{2}(\zeta),\left(\frac{h}{2}\right)^{3} P_{3}(\zeta)\right] \sigma_{\alpha \beta}^{(k)} \mathrm{d} x_{3}
$$

$\left[N_{3 i}, M_{3 i}, K_{3 i}, Z_{3 i}, L_{3 i}\right] \equiv \sum_{k=1}^{N} \int_{A^{(k)}}\left[1, \frac{h}{2} P_{1}(\zeta),(-1)^{k} \frac{2}{n^{(k)} h}\right.$

$$
\left.(-1)^{k} \frac{2}{n^{(k) h}} x_{3}^{(k)},\left(\frac{h}{2}\right)^{2} P_{2}(\zeta)\right] \tau \frac{\tau_{3 i}^{(k)}}{\mathrm{d} x_{3}}
$$

(b) constitutive equations

(1) for transverse stresses

$$
\begin{aligned}
& Q_{\alpha}^{(k)}-\frac{8 J_{\alpha}^{(k)}}{\left(n^{(k)} h\right)^{2}}+ \frac{n^{(k)} h}{30}\left(T_{\alpha}^{(k-1)}+T_{\alpha}^{(k)}\right) \\
&=\frac{2}{5} h n^{(k)} \tilde{c}_{\alpha}^{(k)}\left[U_{3, \alpha}+\Psi_{\alpha}+S_{\alpha}(-1)^{k} \frac{2}{n^{(k)} h}+h n_{0}^{(k)}\left(\Psi_{3, \alpha}+3 \xi_{\alpha}\right)\right. \\
&\left.+\frac{h^{2}}{2}\left(3 n_{0}^{(k) 2}-\frac{1}{4}\right) \xi_{3, \alpha}+\frac{3 h^{2}}{2}\left(5 n_{0}^{(k) 2}-\frac{1}{4}\right) \Phi_{\alpha}\right]
\end{aligned}
$$

$\frac{1}{h} R_{\alpha}^{(k)}-\frac{n^{(k) 2} h}{40}\left(T_{\alpha}^{(k-1)}-T_{\alpha}^{(k)}\right)$

$$
\begin{aligned}
& =\frac{7 h^{2}}{120} n^{(k) 3} \tilde{c}_{\alpha}^{(k)}\left[\Psi_{3, \alpha}+3 \xi_{\alpha}+S_{3, \alpha}(-1)^{k} \frac{2}{n^{(k) h}}+3 h n_{0}^{(k)}\left(\xi_{3, \alpha}+5 \Phi_{\alpha}\right)\right] \\
& Q_{\alpha}^{(k)}-\frac{14 J_{\alpha}^{(k)}}{\left(n^{(k)} h\right)^{2}}+\frac{n^{(k)} h}{12}\left(T_{\alpha}^{(k-1)}+T_{\alpha}^{(k)}\right)=-\frac{3 h^{3}}{40} n^{(k) 3} \bar{c}_{\alpha}^{(k)}\left(\xi_{3, \alpha}+5 \Phi_{\alpha}\right) \\
& -\frac{1}{\tilde{c}_{\alpha}^{(k)}}\left[\frac{1}{12} Q_{\alpha}^{(k)}-\frac{5 J_{\alpha}^{(k)}}{3\left(n^{(k)} h\right)^{2}}+\frac{3 R_{\alpha}^{(k)}}{7 n^{(k)} h}\right]-\frac{1}{\tilde{c}_{\alpha}^{(k+1)}}\left[\frac{1}{12} Q_{\alpha}^{(k+1)}-\frac{5 J_{\alpha}^{(k+1)}}{3\left(n^{(k+1)} h\right)^{2}}-\frac{3 R_{\alpha}^{(k+1)}}{7 n^{(k+1)} h}\right] \\
& =\frac{h}{126}\left[\frac{-n^{(k)}}{\tilde{c}_{\alpha}^{(k)}} T_{\alpha}^{(k-1)}+8\left(\frac{n^{(k)}}{\tilde{c}_{\alpha}^{(k)}}+\frac{n^{(k+1)}}{\tilde{c}_{\alpha}^{(k+1)}}\right) T_{\alpha}^{(k)}-\frac{n^{(k+1)}}{\tilde{c}_{\alpha}^{(k+1)}} T_{\alpha}^{(k+1)}\right]
\end{aligned}
$$

(2) for normal stresses

$$
\begin{aligned}
& Q_{3}^{(k)}-\frac{8 J_{3}^{(k)}}{\left(n^{(k)} h\right)^{2}}+\frac{n^{(k)} h}{30}\left(T_{3}^{(k-1)}+T_{3}^{(k)}\right) \\
&=\frac{2 h}{5} n^{(k)} c_{33}^{(k)}\left[\Psi_{3}+S_{3}(-1)^{k} \frac{2}{n^{(k)} h}+3 h n_{0}^{(k)} \xi_{3}\right]+\frac{2 h}{5} n^{(k)}\left[\tilde{U}+h n_{0}^{(k)} \tilde{\Psi}\right. \\
&\left.+\frac{h^{2}}{2}\left(3 n_{0}^{(k) 2}-\frac{1}{4}\right) \tilde{\xi}+\frac{h^{3}}{2}\left(5 n_{0}^{(k) 3}-\frac{3}{4} n_{0}^{(k)}\right) \tilde{\Phi}\right]
\end{aligned}
$$




$$
\begin{aligned}
& \frac{1}{\mathrm{~h}} R_{3}^{(k)}-\frac{32 I_{3}^{(k)}}{5 n^{(k) 2} h^{3}}+\frac{n^{(k) 2} h}{140}\left(T_{3}^{(k-1)}-T_{3}^{(k)}\right) \\
& =\frac{11}{350} h^{2} n^{(k) 3} c_{33}^{(k)} \xi_{3}+\frac{11}{1050} h^{2} n^{(k) 3}\left[\tilde{\Psi}+(-1)^{k} \frac{2}{n^{(k)} h} \tilde{S}+3 h n_{0}^{(k)} \tilde{\xi}+\frac{3 h^{2}}{2}\left(5 n_{0}^{(k) 2}-\frac{1}{4}\right) \tilde{\Phi}\right] \\
& Q_{3}^{(k)}-\frac{14 J_{3}^{(k)}}{\left(n^{(k)} h\right)^{2}}+\frac{n^{(k)} h}{12}\left(T_{3}^{(k-1)}+T_{3}^{(k)}\right)=-\frac{3 h^{3}}{40} n^{(k) 3}\left[\tilde{\xi}+5 h n_{0}^{(k)} \tilde{\Phi}\right] \\
& \frac{1}{h} R_{3}^{(k)}-\frac{15 I_{3}^{(k)}}{2 n^{(k) 2} h^{3}}+\frac{n^{(k) 2} h}{96}\left(T_{3}^{(k-1)}-T_{3}^{(k)}\right)=-\frac{11 h^{4}}{2688} n^{(k) 5} \tilde{\Phi} \\
& \frac{-11}{12}\left[\frac{Q_{3}^{(k)}}{c_{33}^{(k)}}+\frac{Q_{3}^{(k+1)}}{c_{33}^{(k+1)}}\right]+\frac{15}{2 h}\left[\frac{R_{3}^{(k)}}{n^{(k)} c_{33}^{(k)}}-\frac{R_{3}^{(k+1)}}{n^{(k+1)} c_{33}^{(k+1)}}\right] \\
& +\frac{55}{3 h^{2}}\left[\frac{J_{3}^{(k)}}{n^{(k) 2} c_{33}^{(k)}}+\frac{J_{3}^{(k+1)}}{n^{(k+1) 2} c_{33}^{(k+1)}}\right]-\frac{70}{h^{3}}\left[\frac{I_{3}^{(k)}}{n^{(k) 3} c_{33}^{(k)}}-\frac{I_{3}^{(k+1)}}{n^{(k+1) 3} c_{33}^{(k+1)}}\right] \\
& =\frac{h}{18}\left[\frac{n^{(k)}}{c_{33}^{(k)}} T_{3}^{(k-1)}+10\left(\frac{n^{(k)}}{c_{33}^{(k)}}+\frac{n^{(k+1)}}{c_{33}^{(k+1)}}\right) T_{3}^{(k)}+\frac{n^{(k+1)}}{c_{33}^{(k+1)}} T_{3}^{(k+1)}\right]
\end{aligned}
$$

In eqns (14a-c) and (15a-d), $k$ ranges from 1 to $N$, while in eqns (14d) and (15e), $k$ ranges from 1 to $(N-1)$. Also, no summation on $\alpha$ is implied in eqn (14) and

$$
\begin{aligned}
& \tilde{\mathcal{c}}_{x}^{(k)} \equiv \delta_{\alpha 1} c_{55}^{(k)}+\delta_{\alpha 2} c_{44}^{(k)}, \quad n_{0}^{(k)}=\frac{x_{30}^{(k)}}{h} \\
& {\left[\begin{array}{c}
\tilde{U} \\
\tilde{\Psi} \\
\tilde{S} \\
\tilde{\xi} \\
\tilde{\Phi}
\end{array}\right]=\left[\begin{array}{ll}
U_{1,1} & U_{2,2} \\
\Psi_{1,1} & \Psi_{2,2} \\
S_{1,1} & S_{2,2} \\
\xi_{1,1} & \xi_{2,2} \\
\Phi_{1,1} & \Phi_{2,2}
\end{array}\right]\left[\begin{array}{l}
c_{13} \\
c_{23}
\end{array}\right]^{(k)} .}
\end{aligned}
$$

By solving eqns (14) and (15), $Q_{i}^{(k)}, R_{i}^{(k)}, J_{i}^{(k)}, i_{3}^{(k)}$ and $T_{i}^{(k)}$ are obtained in terms of $U_{i}$, $\Psi_{i}, S_{i}, \xi_{i}$ and $\Phi_{\alpha}$ and their derivatives. As a result, the quantities $N_{3 i}, M_{3 i}, \mathrm{~K}_{3 i}, \mathrm{Z}_{3 i}, L_{3 i}$ of eqn (13b) can be determined as functions of these displacement variables. Such expressions will automatically include the appropriate shear correction factors by virtue of the Reissner mixed variational principle.

The equilibrium equations (12) are supplemented with the following suitable boundary conditions :

$$
\begin{array}{ccc}
\text { specify } U_{i} & \text { or } & N_{\alpha i} v_{\alpha} \\
\text { specify } \Psi_{i} & \text { or } & M_{\alpha i} v_{\alpha} \\
\text { specify } S_{i} & \text { or } & Z_{\alpha i} v_{\alpha} \\
\text { specify } \xi_{i} & \text { or } & L_{\alpha i} v_{\alpha} \\
\text { specify } \Phi_{\alpha} & \text { or } & P_{\beta \alpha} v_{\beta} .
\end{array}
$$

The remaining constitutive equations for $N_{\alpha \beta}, M_{\alpha \beta}, Z_{\alpha \beta}, L_{\alpha \beta}$ and $P_{\alpha \beta}$ are obtained by substituting eqns (1a), (6) and (8b) into eqn (13a) to yield 


$$
\left[\begin{array}{c}
\frac{1}{h} \underset{\sim}{\sim} \\
\frac{1}{h^{2}} \underset{\sim}{\sim} \\
\frac{1}{h} \underset{\sim}{\sim} \\
\frac{1}{h^{3}} \underset{\sim}{\sim} \\
\frac{1}{h^{4}} \underset{\sim}{P}
\end{array}\right]=\left[\begin{array}{ccccc}
{\left[N_{U}\right]} & {\left[N_{\Psi}\right]} & 0 & {\left[N_{\xi}\right.} & {\left[N_{\Phi}\right]} \\
& {\left[M_{\Psi}\right]} & {\left[M_{S}\right]} & {\left[M_{\xi}\right]} & {\left[M_{\Phi}\right]} \\
& & \frac{1}{3}\left[N_{U}\right] & {\left[Z_{\xi}\right]} & {\left[Z_{\Phi}\right]} \\
\text { Symmetric } & & & {\left[L_{\xi}\right]} & {\left[L_{\Phi}\right]} \\
& & & & {\left[P_{\Phi}\right]}
\end{array}\right]\left[\begin{array}{c}
\underset{\sim}{\mathcal{H}} \\
\underset{\sim}{S} \\
h^{2} \underset{\sim}{\xi} \\
h^{3} \underset{\sim}{\Phi}
\end{array}\right]
$$

$$
+i \sum_{k=1}^{N}[C]^{(k)}\left[\begin{array}{c}
V^{N} \\
\underline{V}^{M} \\
\underline{V}^{Z} \\
\tilde{V}^{L} \\
\underline{V}^{P}
\end{array}\right]\left[\begin{array}{c}
Q_{3} \\
\frac{1}{h} R_{3} \\
\frac{1}{h^{2}} J_{3} \\
\frac{1}{h^{3}} I_{3}
\end{array}\right]^{(k)}
$$

where $\underset{\sim}{N}=\left[N_{11}, N_{22}, N_{12}\right]^{T}, \underset{\sim}{U}=\left[U_{1,1}, U_{2,2}, U_{1,2}+U_{2,1}\right]^{T}$ with same expressions for $\underset{\sim}{M}$, $\underline{\Psi} \ldots \underset{\sim}{P} \tilde{\Phi},\left[N_{U}\right] \ldots\left[P_{\phi}\right]$ are $3 \times 3$ matrices, $[C]^{(k)}$ is a $15 \times 5$ matrix and $\underline{\sim}^{N} \ldots V^{P}$ are $1 \times 4$ vectors.

\section{BENDING OF RECTANGULAR LAMINATED PLATES}

The proposed theory can be used to solve the bending problem of rectangular plates for which two opposite edges are simply supported. The other two edges can each have arbitrary boundary conditions. Here we assume that the edges parallel to the $x_{2}$-axis are simply supported, and the origin of the coordinate system is taken as shown in Fig. 2. The simply supported boundary conditions can be satisfied by trigonometric functions in $x_{1}$. The resulting ordinary differential equations in $x_{2}$ can be solved using the state-space concept.

The prescribed boundary conditions on the top and bottom surfaces of the plate are

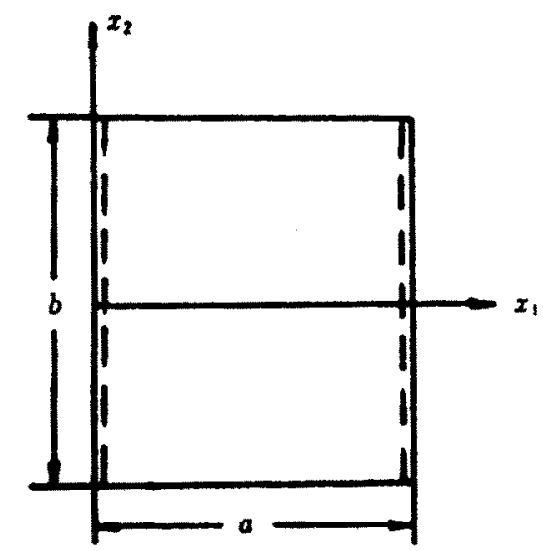

Fig. 2. Geometry and coordinate system of rectangular plate. 


$$
\begin{array}{cl}
T_{1}^{+}=T_{2}^{+}=0, \quad T_{3}^{+}=q & \text { on } x_{3}=\frac{h}{2} \\
T_{1}^{-}=T_{2}^{-}=T_{3}^{-}=0 & \text { on } x_{3}=-\frac{h}{2} .
\end{array}
$$

The following representations of the displacements and loading are assumed:

$$
\begin{gathered}
{\left[\begin{array}{c}
U_{1} \\
\Psi_{1} \\
S_{1} \\
\xi_{1} \\
\Phi_{1}
\end{array}\right]=\sum_{m=1}^{\infty}\left[\begin{array}{c}
h \hat{U}_{1 m}\left(x_{2}\right) \\
\hat{\Psi}_{1 m}\left(x_{2}\right) \\
h \hat{S}_{1 m}\left(x_{2}\right) \\
\frac{1}{h} \hat{\xi}_{1 m}\left(x_{2}\right) \\
\frac{1}{h^{2}} \hat{\Phi}_{1 m}\left(x_{2}\right)
\end{array}\right] \cos \alpha x_{1}\left[\begin{array}{c}
U_{2} \\
\Psi_{2} \\
S_{2} \\
\xi_{2} \\
\Phi_{2}
\end{array}\right]=\sum_{m=1}^{\infty}\left[\begin{array}{c}
h \hat{U}_{2 m}\left(x_{2}\right) \\
\hat{\Psi}_{2 m}\left(x_{2}\right) \\
h \hat{S}_{2 m}\left(x_{2}\right) \\
\frac{1}{h} \hat{\xi}_{2 m}\left(x_{2}\right) \\
\frac{1}{h^{2}} \hat{\Phi}_{2 m}\left(x_{2}\right)
\end{array}\right] \sin \alpha x_{1}} \\
{\left[\begin{array}{c}
U_{3} \\
\Psi_{3} \\
S_{3} \\
\xi_{3}
\end{array}\right]=\sum_{m=1}^{\infty}\left[\begin{array}{c}
h \hat{U}_{3 m}\left(x_{2}\right) \\
\hat{\Psi}_{3 m}\left(\mathrm{x}_{2}\right) \\
h \hat{S}_{3 m}\left(x_{2}\right) \\
\frac{1}{h} \hat{\xi}_{3 m}\left(x_{2}\right)
\end{array}\right] \sin \alpha x_{1}} \\
q=\sum_{m=1}^{\infty} Q_{m}\left(x_{2}\right) \sin \alpha \mathrm{x}_{1},
\end{gathered}
$$

where $\alpha=m \pi / a$ and $\hat{U}_{1 m} \ldots \hat{\xi}_{3 m}$ and $Q_{m}$ denote amplitudes of $U_{1} \ldots \xi_{3}$ and $q$, respectively. It is easily proven that eqn (21) can satisfy boundary condition of simply supported on $x_{1}=0, a$ i.e. at $x_{1}=0, a$

$$
U_{3}=\Psi_{3}=S_{3}=\zeta_{3}=0 \text { and } N_{11}=M_{11}=Z_{11}=L_{11}=P_{11}=0
$$

Then inserting eqn (21) into constitutive equations and these with eqn (22) in turn into the equilibrium eqn (12), yields a system of fourteen ordinary differential equations in the $x_{2}$ coordinate, which can be reduced to a single matrix differential equation using the statespace concept (Franklin, 1986)

$$
X^{\prime}=A X+B
$$

This can be done by introducing the variables

$$
X=\left[\hat{U}_{1 m}^{\prime} \hat{U}_{1 m} \hat{\Psi}_{1 m}^{\prime} \hat{\Psi}_{1 m} \hat{S}_{1 m}^{\prime} \hat{S}_{1 m} \hat{\xi}_{1 m}^{\prime} \hat{\xi}_{1 m} \hat{\Phi}_{1 m}^{\prime} \hat{\Phi}_{1 m} \hat{U}_{2 m}^{\prime} \hat{U}_{2 m}^{\prime} \ldots \hat{\Phi}_{2 m}^{\prime} \hat{\Phi}_{2 m} \hat{U}_{3 m}^{\prime} \hat{U}_{3 m} \ldots \hat{\xi}_{3 m}^{\prime} \hat{\xi}_{3 m}\right]^{T}
$$

where $A$ is a $28 \times 28$ matrix which depends on the volume fractions $n^{(k)}$ and elastic constants $\bar{c}_{i j}$ and $B$ is a $1 \times 28$ vector which depends on $Q_{m}$.

The solution of eqn (24) is given by

$$
X=\mathrm{e}^{A x_{2}} K+\mathrm{e}^{A x_{2}} \int_{-b ; 2}^{x_{2}} \mathrm{e}^{-A \eta} B \mathrm{~d} \eta,
$$

where $K$ is a $1 \times 28$ constant vector to be determined from the boundary conditions, while $\mathrm{e}^{A x_{2}}$ is defined by 


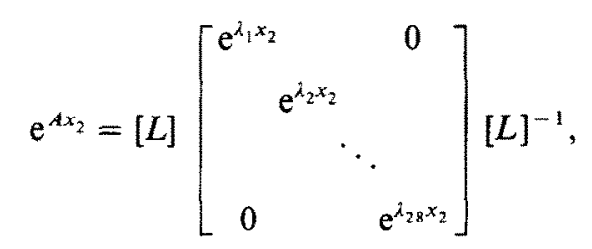

where $[L]$ is the matrix of eigenvectors, $\lambda_{i}(i=1,2 \ldots 28)$ denote the distinct eigenvalues associated with the matrix $A$ and $[L]^{-1}$ is the inverse of the matrix $[L]$.

The following boundary conditions are used on the remaining two edges (i.e. the edges parallel to the $x_{1}$-axis) at $x_{2}-1 b / 2$ :

simply supported

$$
\begin{aligned}
U_{1} & =\Psi_{1}=S_{1}=\xi_{1}=\Phi_{1}=0 \\
U_{3} & =\Psi_{3}=S_{3}=\xi_{3}=0 \\
N_{22} & =M_{22}=Z_{22}=L_{22}=P_{22}=0
\end{aligned}
$$

clamped

$$
\begin{aligned}
& U_{1}=\Psi_{1}=S_{1}=\xi_{1}=\Phi_{1}=0 \\
& U_{2}=\Psi_{2}=S_{2}=\xi_{2}=\Phi_{2}=0 \\
& U_{3}=\Psi_{3}=S_{3}=\xi_{3}=0
\end{aligned}
$$

free

$$
\begin{aligned}
& N_{12}=N_{22}=N_{23}=0 \\
& M_{12}=M_{22}=M_{23}=0 \\
& Z_{12}=Z_{22}=Z_{23}=0 \\
& L_{12}=L_{22}=L_{23}=0 \\
& P_{12}=P_{22}=0 .
\end{aligned}
$$

\section{NUMERICAL RESULTS AND DISCUSSION}

The following numerical examples are presented:

(a) Numerical results are presented for orthotropic and symmetric cross-ply $\left(0^{\circ} / 90^{\circ} / 0^{\circ}\right)$ plate with same thickness layer subject to three types of loads; uniformly distributed load $\left(q_{0}\right)$, triangular distributed load $\left(2 q_{0}\right)$ and concentrated load $p$, as shown in Fig. 3.

The following dimensionless orthotropic matcrial properties are used:

$$
\begin{array}{ccc}
\frac{E_{1}}{E_{0}}=20.83 & \frac{E_{2}}{E_{0}}=10.94 & E_{3}=E_{2} \\
\frac{G_{12}}{E_{0}}=6.10 & \frac{G_{13}}{E_{0}}=3.71 & \frac{G_{23}}{E_{0}}=6.19 \\
v_{12}=v_{13}=v_{23}=0.44 & E_{0}=1 \times 10^{6} \mathrm{psi} .
\end{array}
$$
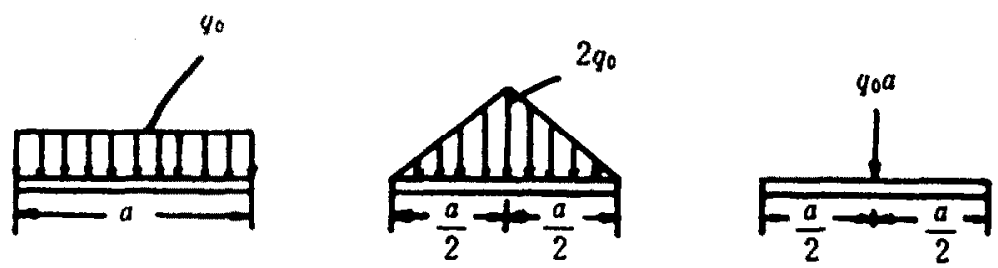

Fig. 3. Various types of transverse loads. 
All results are compared with Khdeir's solution (Khdeir et al., 1987) as shown in Tables 1-3. Tables 1-2 contain center deflections $\bar{u}_{3}$ for orthotropic and symmetric crossply $\left(0^{\circ} / 90^{\circ} / 0^{\circ}\right)$ plates, while Table 3 contains non-dimensionalized axial stresses $\bar{\sigma}_{11}$ for cross-ply $\left(0^{\circ} / 90^{\circ} / 0^{\circ}\right)$ plate.

The following notation has been used throughout the tables:

SS simply supported at $x_{2}=-b / 2$ and at $x_{2}=b / 2$;

CC clamped at $x_{2}=-b / 2$ and at $x_{2}=b / 2$;

FF free at $x_{2}=-b / 2$ and at $x_{2}=b / 2$;

$\mathrm{SC}$ simply supported at $x_{2}=-b / 2$ and clamped at $x_{2}=b / 2$;

SF simply supported at $x_{2}=-b / 2$ and free at $x_{2}=b / 2$;

$\mathrm{CF}$ clamped at $x_{2}=-b / 2$ and free at $x_{2}=b / 2$;

UN uniformly distributed load;

TR triangular distributed load;

PL point load at the center of the plate.

Figure 4 shows the thickness variations of axial stresses $\sigma_{11}$ of $\left(0^{\circ} / 90^{\circ} / 0^{\circ}\right)$ laminated plate for various boundary conditions.

(b) Center deflections and stresses for cross-ply plates under sinusoidal transverse loading

$$
\text { (i.e. } \left.q=q_{0} \sin \frac{\pi}{a} x_{1} \cos \frac{\pi}{b} x_{2}\right)
$$

are calculated. The plates are simply supported at four edges. The numerical results are compared with exact elastic solution obtained by Pagano (1970).

The following material properties are used:

$$
\begin{array}{ccc}
\frac{E_{1}}{E_{0}}=25 & \frac{E_{2}}{E_{0}}=1.0 & E_{3}=E_{2} \\
\frac{G_{12}}{E_{0}}=0.5 & G_{13}=G_{12} & \frac{G_{23}}{E_{0}}=0.2 \\
v_{12}=v_{13}=v_{23}=0.25 & E_{0}=1 \times 10^{6} \text { psi. }
\end{array}
$$

We follow Pagano's non-dimensionalization and write the center deflection and stresses in the form:

$$
\begin{array}{ll}
\bar{u}_{3}=\frac{100 E_{0} h^{3}}{q_{0} a^{4}} u_{3}\left(\frac{a}{2}, 0,0\right) & \\
\bar{\sigma}_{11}=\frac{h^{2}}{q_{0} a^{2}} \sigma_{11}\left(\frac{a}{2}, 0, x_{3}\right), & \bar{\sigma}_{22}=\frac{h^{2}}{q_{0} a^{2}} \sigma_{22}\left(\frac{a}{2}, 0, x_{3}\right) \\
\bar{\tau}_{12}=\frac{h^{2}}{q_{0} a^{2}} \tau_{12}\left(0,-\frac{b}{2}, x_{3}\right), & \bar{\tau}_{23}=\frac{h}{q_{0} a} \tau_{23}\left(\frac{a}{2},-\frac{b}{2}, x_{3}\right) \\
\bar{\tau}_{31}=\frac{h}{q_{0} a} \tau_{31}\left(0,0, x_{3}\right), & \bar{\sigma}_{33}=\frac{h}{q_{0} a} \sigma_{33}\left(\frac{a}{2}, 0, x_{3}\right) .
\end{array}
$$

Also

$$
\bar{x}_{3}=x_{3} / h \quad S=a / h .
$$

Tables 4-6 show the center deflections and in-plane stresses and transverse shear stresses of the various side-to-thickness ratios for cross-ply rectangular plate $\left(0^{\circ} / 90^{\circ} / 0^{\circ}\right.$, 
Bending solution of high-order refined shear deformation theory

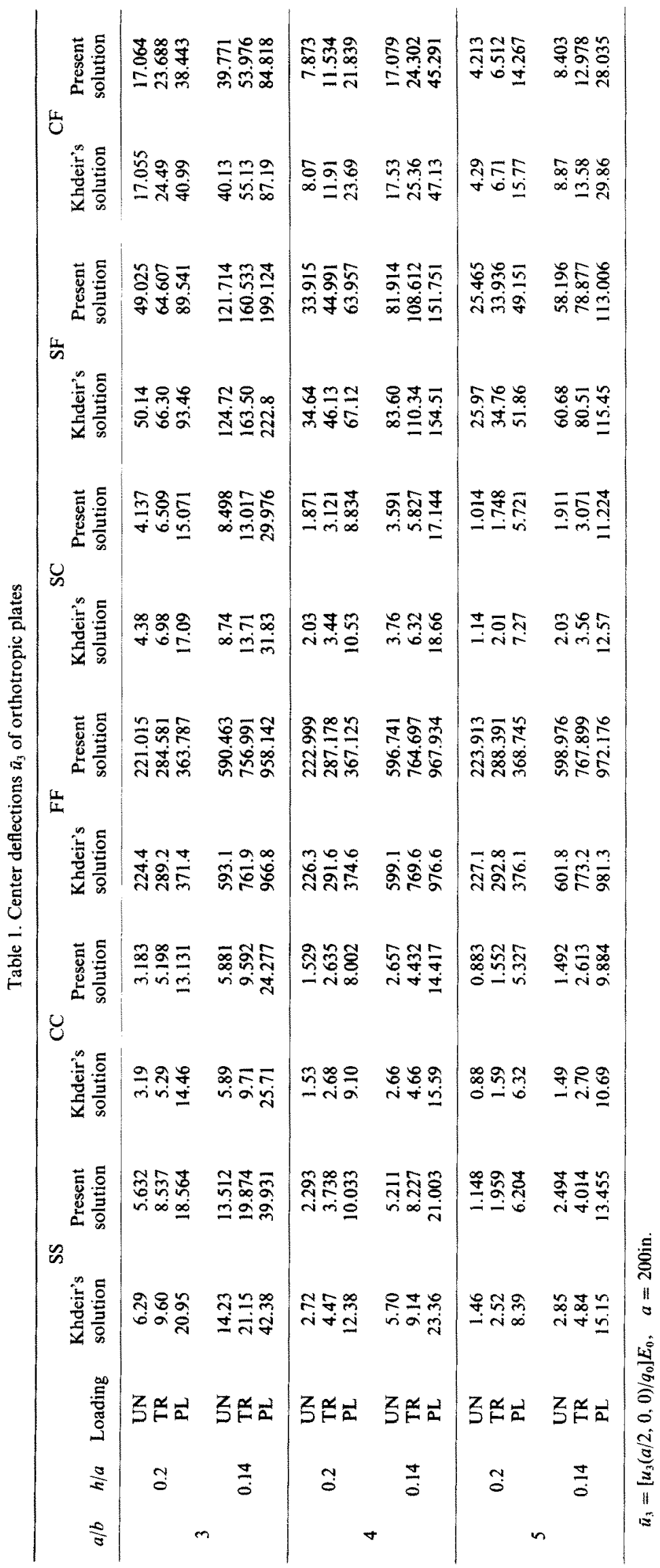




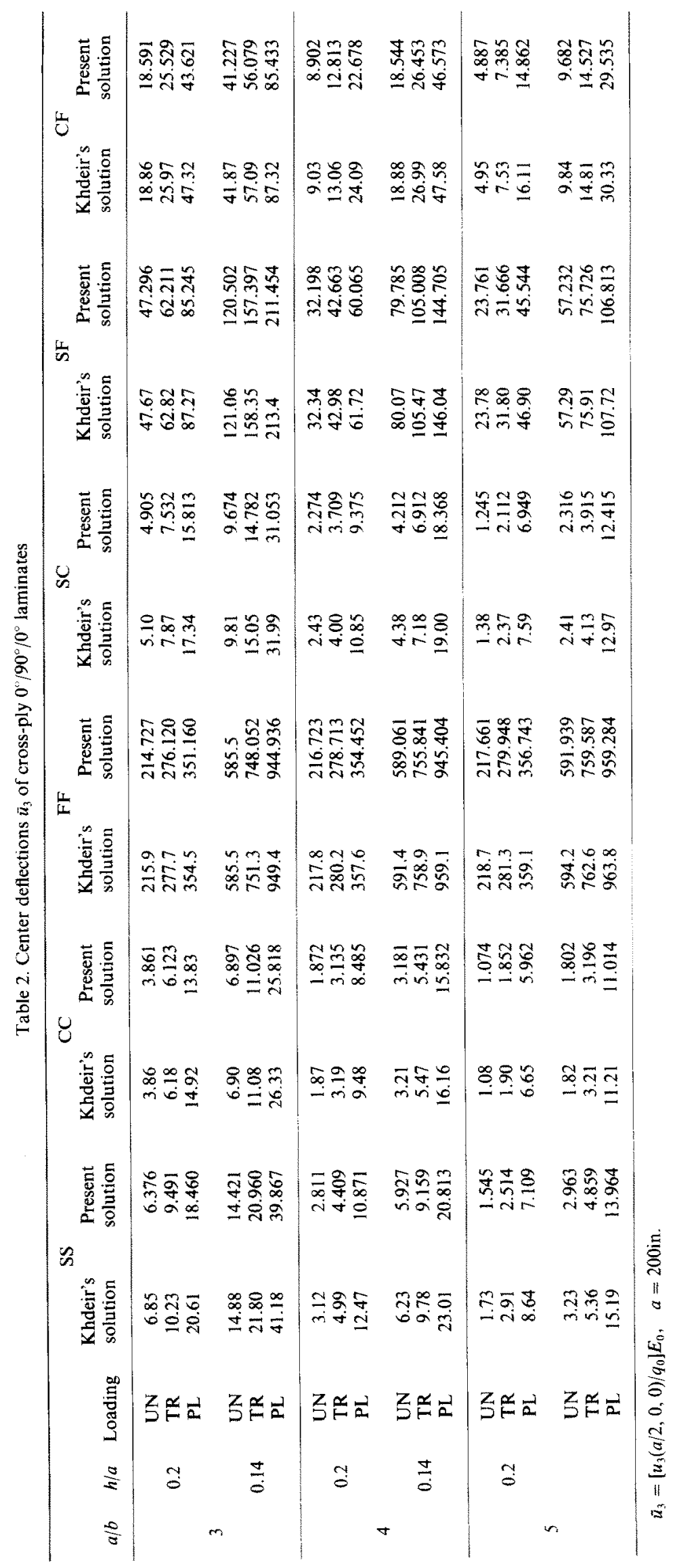




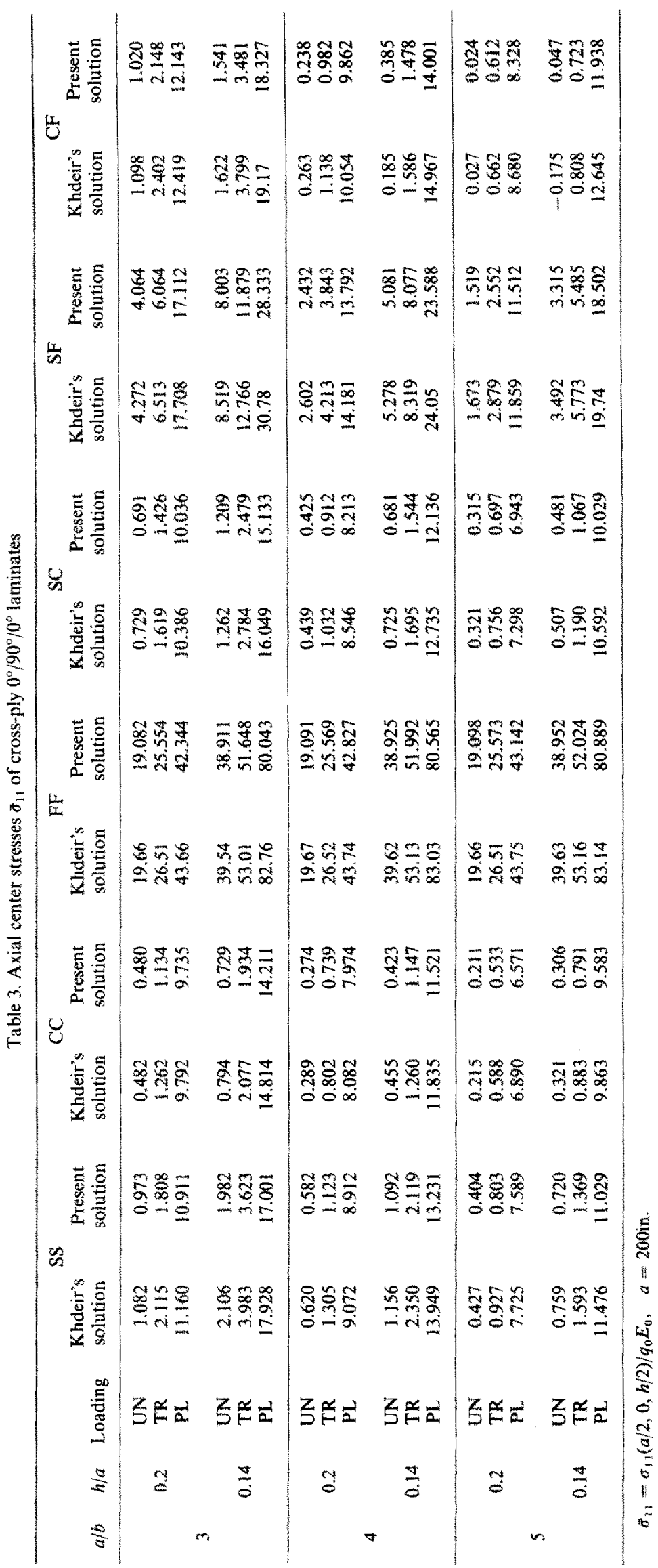




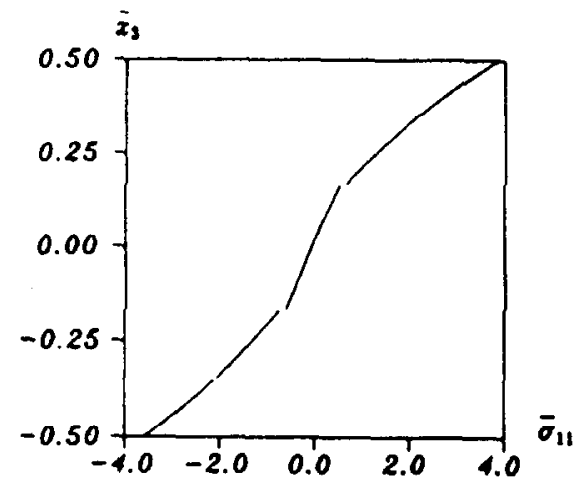

(a)

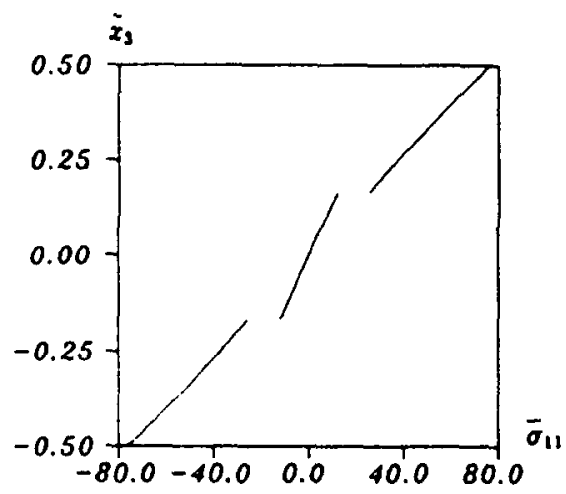

(c)

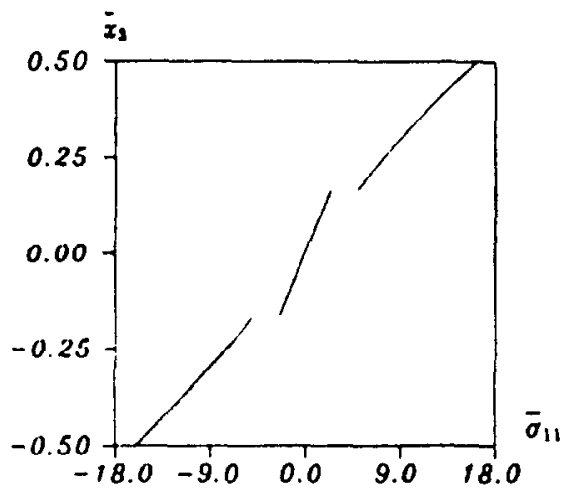

(e)

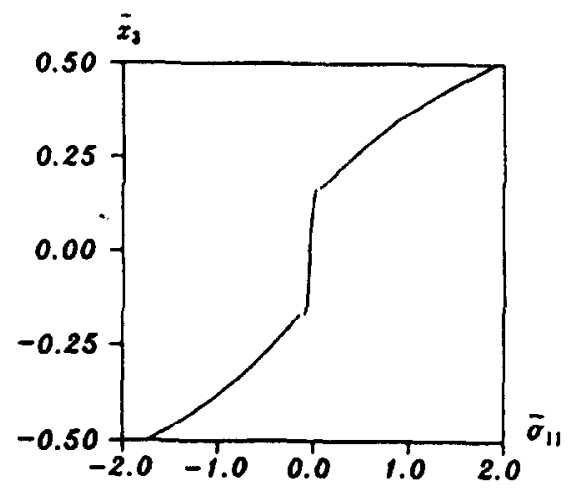

(b)

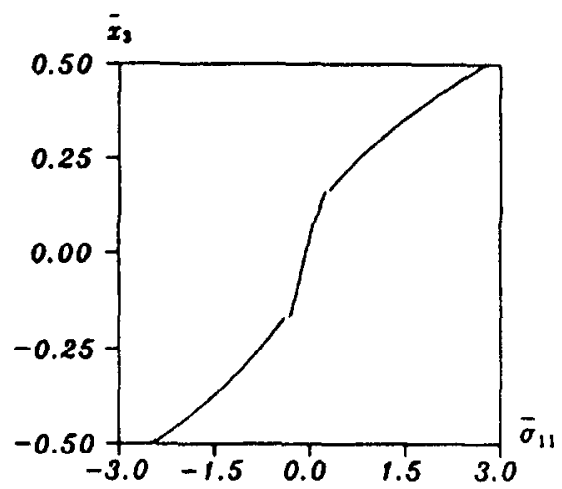

(d)

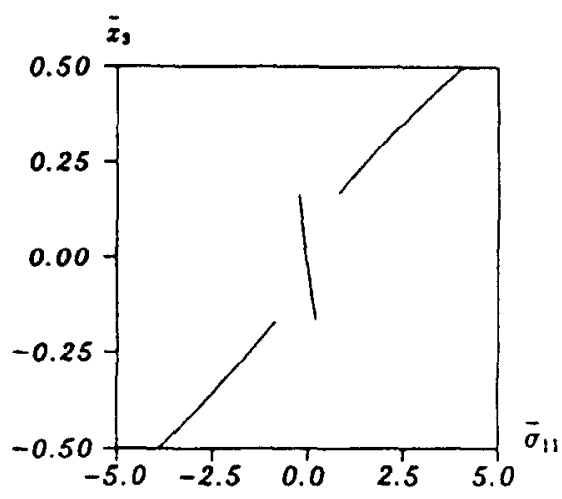

(f)

Fig. 4. Thickness variations of $\sigma_{11}$ for various boundary conditions $(a / b=3, a / h=5, \mathrm{UN})$ : (a)-SSSS ; (b)-SSCC; (c) -SSFF; (d) -SSSC; (e)-SSSF; (f) -SSCF.

$b / a=3)$ and square plates $\left[0^{\circ} / 90^{\circ} / 90^{\circ} / 0^{\circ}, 0^{\circ} / 90^{\circ} \ldots 0^{\circ}\right.$ (9 layers)] with the same thickness layer, respectively.

For the side-to-thickness ratio $S=0$, the thickness variations of in-plane and transverse shear and normal stresses for $0^{\circ} / 90^{\circ} / 0^{\circ}$ laminated plate are shown in Fig. 
Table 4. Center deflections and stresses of $0^{\circ} / 90^{\circ} / 0^{\circ}$ laminates

\begin{tabular}{clcccccc}
\hline$S$ & Theory & $\bar{u}_{3}$ & $\sigma_{11}\left(\frac{h}{2}\right)$ & $\sigma_{22}\left(\frac{h}{6}\right)$ & $\sigma_{23}(0)$ & $\sigma_{11}(0)$ & $\sigma_{12}\left(\frac{h}{2}\right)$ \\
\hline & $\begin{array}{l}\text { Pagano's } \\
\text { solution }\end{array}$ & 2.82 & 1.14 & 0.109 & 0.0334 & 0.351 & -0.0269 \\
\hline $\begin{array}{l}\text { Present } \\
\text { solution }\end{array}$ & 2.8234 & 1.1242 & 0.108 & 0.03276 & 0.3558 & -0.02748 \\
\hline $\begin{array}{l}\text { Pagano's } \\
\text { solution }\end{array}$ & 0.919 & 0.726 & 0.0418 & 0.0152 & 0.420 & -0.0120 \\
\hline $\begin{array}{l}\text { Present } \\
\text { solution }\end{array}$ & 0.9148 & 0.7193 & 0.04152 & 0.01591 & 0.4143 & -0.01204 \\
\hline $\begin{array}{l}\text { Pagano's } \\
\text { solution }\end{array}$ & 0.610 & 0.650 & 0.0299 & 0.0119 & 0.434 & -0.0093 \\
$\begin{array}{l}\text { Present } \\
\text { solution } \\
\text { Pagano's }\end{array}$ & 0.6047 & 0.6439 & 0.02921 & 0.01282 & 0.4410 & -0.0092 \\
\hline solution & 0.508 & 0.624 & 0.0253 & 0.0108 & 0.439 & -0.0083 \\
\hline $\begin{array}{l}\text { Present } \\
\text { solution }\end{array}$ & 0.5029 & 0.6185 & 0.02507 & 0.0118 & 0.4459 & -0.00824 \\
\hline
\end{tabular}

From Tables 1-3, it is shown that the center deflections and stresses are slightly smaller than those obtained by Khdeir. This is because the present theory includes the effect of transverse normal strain $\left(\varepsilon_{33}\right)$ and stress $\left(\sigma_{33}\right)$. While this theory can satisfy the continuity condition of transverse shear stresses at the interfaces, this is not true for Khdeir's theory.

From Tables 4-6, close agreement for the center deflections and stresses of the present theory and the exact solution obtained by Pagano are observed for different side-tothickness ratio and lamination schemes, which proves that the displacement field and trial transverse and normal stresses field of the present theory are appropriate and reasonable.

In the present work, transverse shear stresses $\tau_{31}$ and $\tau_{32}$ and transverse normal stress $\tau_{33}$ are obtained from eqn (8), which satisfy the top and bottom surfaces boundary

Table 5. Center deflections and stresses of $0^{\circ} / 90^{\circ} / 90^{\circ} / 0^{\circ}$ laminates

\begin{tabular}{cccccccc}
\hline$S$ & Theory & $\bar{u}_{3}$ & $\sigma_{11}\left(\frac{h}{2}\right)$ & $\sigma_{22}\left(\frac{h}{4}\right)$ & $\sigma_{23}(0)$ & $\sigma_{31}(0)$ & $\sigma_{12}\left(\frac{h}{2}\right)$ \\
\hline & $\begin{array}{l}\text { Pagano's } \\
\text { solution }\end{array}$ & 1.954 & 0.720 & 0.663 & 0.292 & 0.291 & -0.0467 \\
\hline $\begin{array}{l}\text { Present } \\
\text { solution }\end{array}$ & 1.884 & 0.7364 & 0.5908 & 0.2343 & 0.2285 & -0.04612 \\
10 & 0.743 & 0.559 & 0.401 & 0.196 & 0.301 & -0.0275 \\
$\begin{array}{l}\text { Pagano's } \\
\text { solution }\end{array}$ & $\begin{array}{l}\text { Present } \\
\text { solution }\end{array}$ & 0.7097 & 0.5499 & 0.3813 & 0.1548 & 0.3085 & -0.02678 \\
\hline $\begin{array}{l}\text { Pagano's } \\
\text { solution }\end{array}$ & 0.517 & 0.543 & 0.308 & 0.156 & 0.328 & -0.0230 \\
\hline $\begin{array}{l}\text { Present } \\
\text { solution } \\
\text { Pagano's }\end{array}$ & 0.4980 & 0.5315 & 0.2984 & 0.1245 & 0.3340 & -0.02246 \\
\hline $\begin{array}{l}\text { solution } \\
\text { Present }\end{array}$ & 0.4385 & 0.539 & 0.276 & 0.141 & 0.337 & -0.0216 \\
\hline $\begin{array}{l}\text { Polution } \\
\text { soln }\end{array}$ & 0.4247 & 0.5267 & 0.2648 & 0.1123 & 0.3440 & -0.02087 \\
\hline
\end{tabular}




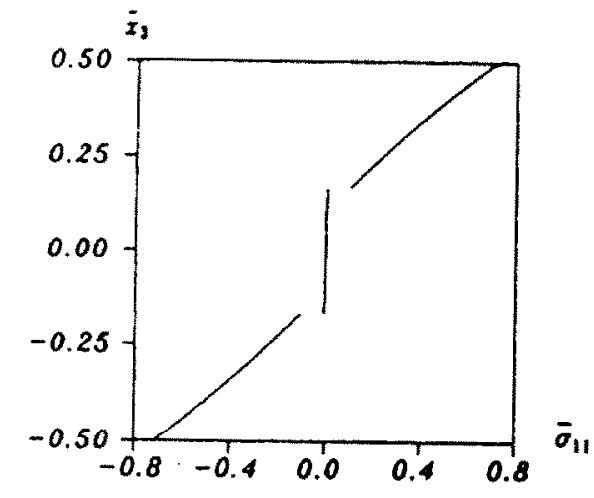

Liu Ping et al.
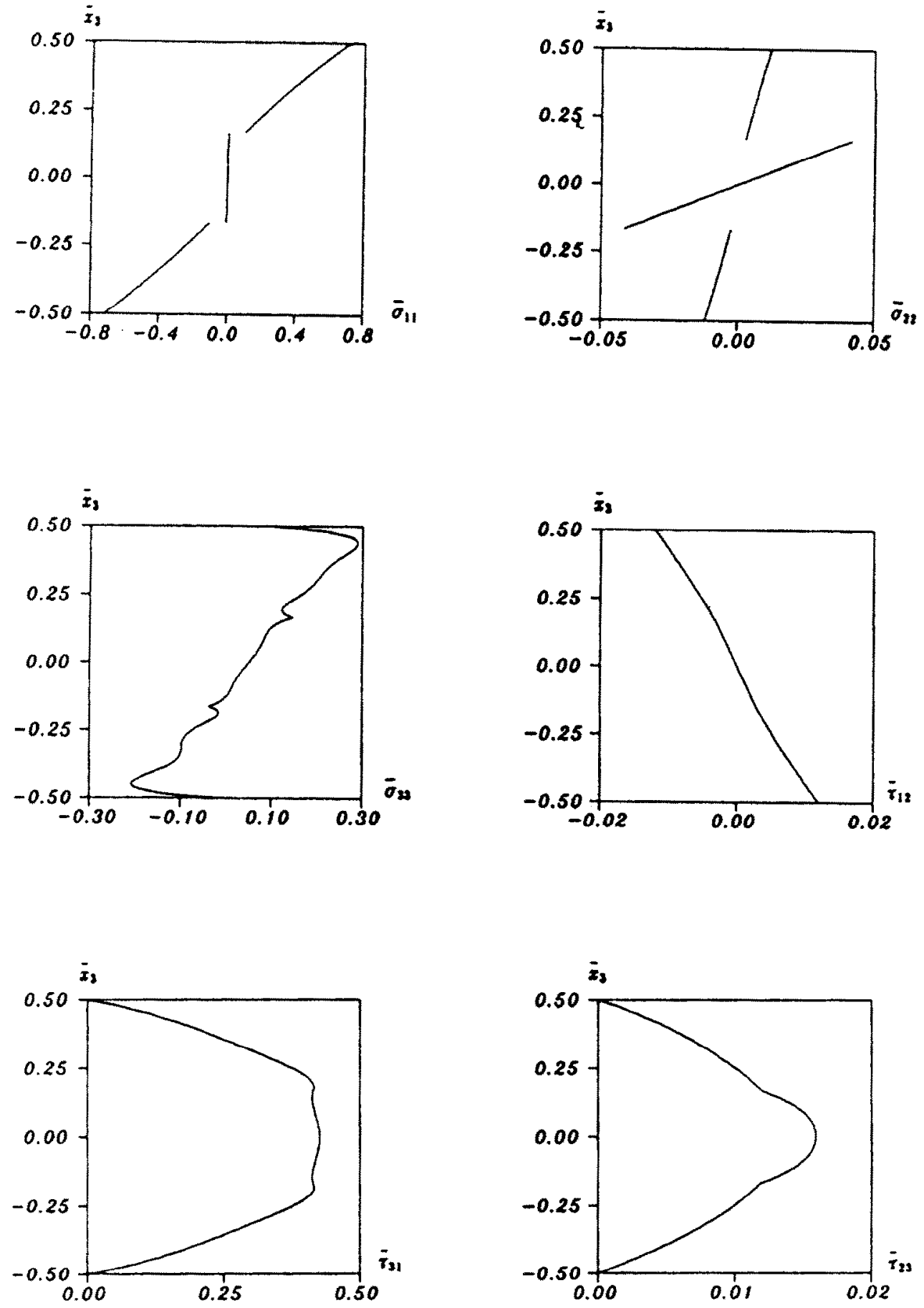

Fig. 5. Thickness variations of stresses.

conditions prescribed by eqn (20) (also see Fig. 5). Reddy (1984a) pointed out that the alternate procedure of computing the transverse stresses by integrating the equilibrium equations using the in-plane stresses found directly from the displacement solution yields more accurate results.

The first-order zig-zag theory proposed by Murakami (1986) is a particular case of this present theory. The author has applied it to the bending problem of rectangular plates. For $0^{\circ} / 90^{\circ} / 0^{\circ}$ plate, better results were obtained, but for $0^{\circ} / 90^{\circ} / 0^{\circ} / 90^{\circ}$ and $0^{\circ} / 90^{\circ} / 90^{\circ} / 0^{\circ}$ plates, the first-order zig-zag theory deviates significantly from the exact solution. However, the present theory is still very good when compared with the exact solution. Obviously, the 
Table 6. Center deflections and stresses of $0^{\circ} / 90^{\circ} \ldots 0^{\circ}$ ( 9 layers) laminates

\begin{tabular}{cccccccc}
\hline$S$ & Theory & $\bar{u}_{3}$ & $\sigma_{11}\left(\frac{h}{2}\right)$ & $\sigma_{22}\left(\frac{2 h}{5}\right)$ & $\sigma_{23}(0)$ & $\sigma_{31}(0)$ & $\sigma_{12}\left(\frac{h}{2}\right)$ \\
\hline & $\begin{array}{l}\text { Pagano's } \\
\text { solution }\end{array}$ & 1.7590 & 0.684 & 0.203 & 0.223 & -0.0337 \\
\hline $\begin{array}{l}\text { Present } \\
\text { solution } \\
\text { Pagano's } \\
\text { solution }\end{array}$ & 1.7501 & 0.6620 & 0.02946 & 0.1990 & 0.2458 & -0.03333 \\
10 & 0.6520 & 0.551 & 0.226 & 0.247 & -0.0233 \\
\hline $\begin{array}{l}\text { Present } \\
\text { solution }\end{array}$ & 0.6409 & 0.5341 & 0.02286 & 0.1878 & 0.2773 & -0.02296 \\
\hline
\end{tabular}

present theory is suitable for arbitrary laminated configurations, so it is the development of the first-order zig-zag theory.

\section{CONCLUSION}

An improved high-order shear deformation theory based upon Reissner's mixed variational principle in conjunction with the state-space concept is developed to determine the bending problems for rectangular laminated composite plate. Numerical results are presented for different edge conditions, aspect ratios, lamination schemes and loadings and are compared with Khdeir and Pagano's theories. The comparison indicates that the present theory accurately estimates in-plane responses, even for small side-to-thickness ratios and large layer laminates.

Acknowledgement-The support of the Natural Science Foundation of China is gratefully acknowledged.

\section{REFERENCES}

Bert, C. W. and Chen, T. L. C. (1978). Effect of shear deformation on vibration of antisymmetric angle-ply laminated rectangular plates. Int. J. Solids Structures 14, 465-473.

Franklin, J. N. (1986). Matrix Theory. Prentice-Hall, Englewood Cliffs, New Jersey.

Hussainy, S. A. and Srinivas, S. (1975). Flexure of rectangular composite plates. Fibre Sci. Technol. 8, 59-76.

Khdeir, A. A., Reddy, J. N. and Librescu, L. (1987). Analytical solution of a refined shear deformation theory for rectangular composite plates. Int. J. Solids Structures 23, 1447-1463.

Murakami, H. (1986). A laminated composite plate theory with improved in-plane responses. ASME J. Appl. Mech. 53, 661-666.

Pagano, N. J. (1970). Exact solutions for rectangular bidirectional composites and sandwich plates. J. Composite Mater. 4, 20-34.

Reddy, J. N. (1984a). A simple higher-order theory for laminated plates. J. Appl. Mech. 51, 745-752.

Reddy, J. N. (1984b). A refined nonlinear theory of plates with transverse shear deformation. Int. J. Solids Structures 20, 881-896.

Reddy, J. N. and Chao, C. W. (1981). A comparison of closed form and finite element solutions of thick laminated anisotropic rectangular plates. Nucl. Engng Des. 64, 153-167.

Reddy, J. N. and Phan, N. D. (1985). Stability and vibration of isotropic, orthotropic and laminated plates according to a higher-order shear deformation theory. J. Sound Vibr. 98(2), 157-170.

Reddy, J. N., Khdeir, A. A. and Librescu, L. (1987). The Lévy type solutions for symmetric rectangular composite plates using the first-order shear deformation theory. J. Appl. Mech. 54, 740-742.

Reissner, E. (1984). On a certain mixed variational theorem and a proposed application. Int. J. Num. Mech. Engng 20, 1366-1368.

Srinivas, S. and Rao, A. K. (1970). Bending, vibration and buckling of simply supported thick orthotropic rectangular plates and laminates. Int. J. Solids Structures 6, 1463-1481.

Srinivas, S., Joga, C. V. and Rao, A. K. (1970). An exact analysis for vibration of simply supported homogeneous and laminated thick rectangular plates. J. Sound Vibr. 12, 187-199.

Whitney, J. M. and Leissa, A. W. (1969). Analysis of heterogeneous anisotropic plates. J. Appl. Mech. 36, 261266.

Whitncy, J. M. and Pagano, N. J. (1970). Shear deformation in heterogeneous anisotropic plates. J. Appl. Mech. 37, 1031-1036. 